\title{
CONJUGATE HOLOMORPHIC EIGENFUNCTIONS AND EXTREMAL QUASICONFORMAL REFLECTION
}

\author{
JAN G. KRZYZ்
}

\section{Introduction}

Let $\Gamma$ be a Jordan curve in the extended plane $\hat{C}$ and let $D$ and $D^{*}$ be its complementary domains. We say that $\Gamma$ is a $K$-quasicircle if it is the image curve of a circle under a $K$-quasiconformal (abbreviated: $K$-qc) mapping of $\hat{\boldsymbol{C}}$ onto itself.

A sense-reversing $K$-qc mapping $\Phi$ of $D$ onto $D^{*}$ whose extension on the closure $\bar{D}$ keeps the points of $\Gamma$ fixed, is called a $K$-qc reflection in $\Gamma$. Obviously $\Phi$ can be extended to a sense-reversing $K$-qc selfmapping of $\hat{\boldsymbol{C}}$ which is defined as $\Phi^{-1}$ in $D^{*}$. A Jordan curve $\Gamma$ admits a $K$-qc reflection if and only if it is a quasicircle; cf. [10], [9].

Various definitions and many interesting properties of quasicircles and quasidisks (i.e., Jordan domains bounded by a quasicircle) are presented in [3].

For a fixed $K>1$ sufficiently large all $K$-qc reflections in a quasicircle $\Gamma$ form a non-empty normal family. Consequently, there exists an extremal qc reflection $\varphi$ in $\Gamma$, i.e., a reflection whose maximal dilatation is a minimum.

In Section 2 we introduce (Definition 1) the notion of conjugate holomorphic eigenfunctions (abbreviated: CHE), which leads to a generalization of the Fredholm eigenvalues of a Jordan curve $\Gamma$ (Definition 2). An additional assumption of the local univalence of $\mathrm{CHE}$ permits us to construct in Section 3 explicilly in terms of $\mathrm{CHE}$ and the associated eigenvalue $\lambda>1$ a $K$-qc reflection $\varphi$ in $\Gamma$ with $K=$ $(\lambda+1) /(\lambda-1)$ which is unique extremal, and the eigenvalue $\lambda$ shows to be the smallest one. In case of classical Fredholm eigenvalues and univalent $\mathrm{CHE}$ our construction implies Theorem 7 in [8].

The results of this paper were presented at a Colloquium in Halle (GDR) on September 29, 1983 and also at the Oberwolfach Conference on February 15, 1984. The author wishes to thank Professor Kühnau for discussions and critical remarks. 


\section{Conjugate holomorphic eigenfunctions}

In what follows we assume that $\Gamma$ is a Jordan curve in the finite plane $C$ and $\infty \in D^{*}$. Many important problems in conformal mappings and the potential theory can be reduced to the solution of a linear integral equation of the Fredholm type with the Neumann kernel (or its transposition):

$$
k(\zeta, t)=-\frac{1}{\pi} \frac{\partial}{\partial n_{\zeta}} \log |\zeta-t|, \quad \zeta, t \in \Gamma,
$$

where $\partial / \partial n_{\zeta}$ denotes the derivative along the interior normal of $\Gamma$ at the point $\zeta$. For details see e.g. [2], [5], [12].

If $\Gamma \in C^{3}$ and $\varkappa(t)$ denotes the curvature of $\Gamma$, then putting $2 \pi k(t, t)=\varkappa(t)$ we obtain a kernel continuously differentiable w.r.t. the arc length $s$ on $\Gamma$. The eigenvalues of $k$, i.e., the real numbers $\lambda$ such that the homogeneous equation

$$
\mu(t)=\lambda \int_{\Gamma} k(\zeta, t) \mu(\zeta) d s, \quad t \in \Gamma,
$$

has a non-trivial real-valued solution $\mu$, are called Fredholm eigenvalues of $\Gamma$.

If $\tau(\zeta)$ is real-valued and continuously differentiable w.r.t. $s$ on $\Gamma \in C^{3}$, then the integral

$$
I(z)=\frac{1}{\pi i} \int_{\Gamma} \frac{\tau(\zeta) d \zeta}{\zeta-z}=\frac{1}{\pi i} \int_{\Gamma} \tau(\zeta) d_{\zeta} \log (\zeta-z)
$$

represents in $D$ and $D^{*}$ holomorphic functions $f$ and $F$, respectively, which for obvious reasons may be called conjugate holomorphic potentials of double layer with density $\tau$. The principal value p.v. $I(t)$ of (2.3) at $t \in \Gamma$ is then a continuous function $\alpha(t)+i \beta(t)$ of $t$ on $\Gamma$, and by the well-known Privalov theorem on Cauchy type integrals (cf. [4]) holomorphic potentials $f, F$ of double layer have continuous extensions on $\bar{D}$ and $\overline{D^{*}}$, respectively, which satisfy on $\Gamma$

$$
f(\zeta)=\alpha(\zeta)+\tau(\zeta)+i \beta(\zeta), \quad F(\zeta)=\alpha(\zeta)-\tau(\zeta)+i \beta(\zeta) .
$$

Following Schiffer [14] we may consider holomorphic potentials of double layer with density $\tau=\lambda \mu$, where $\mu$ is an eigenfunction associated with the eigenvalue $\lambda$. In our case $\mu$ is continuously differentiable w.r.t. $s$. This follows from the fact that $\mu(\zeta(s))$ is a finite linear combination of $D\left(s, t_{k} ; \lambda\right)$, where $D$ is Fredholm's first minor; cf. [11], p. 43, and [5], p. 235. Since for $t \in \Gamma$ we have

$$
\operatorname{Re} I(t)=\int_{\Gamma} \tau(\zeta) k(\zeta, t) d s=\lambda \int_{\Gamma} \mu(\zeta) k(\zeta, t) d s=\mu(t)
$$

and consequently

$$
\text { p.v. } I(\zeta)=\mu(\zeta)+i v(\zeta), \quad \zeta \in \Gamma,
$$

the equations (2.4) take the form

$$
f(\zeta)=(1+\lambda) \mu(\zeta)+i v(\zeta), \quad F(\zeta)=(1-\lambda) \mu(\zeta)+i v(\zeta),
$$


where $\mu, v$ are real-valued and continuous on $\Gamma$. The relations (2.6) can be written in the following concise form:

$$
f(\zeta)=L \circ F(\zeta), \quad F(\zeta)=l \circ f(\zeta)
$$

where

$$
L(w)=(1-\lambda)^{-1}(w+\lambda \bar{w}), \quad l(w)=(1+\lambda)^{-1}(w-\lambda \bar{w})=L^{-1}(w) .
$$

If (2.6) holds, then

$$
[f(\zeta)-F(\zeta)] /[\overline{F(\zeta)}+f(\zeta)]=\lambda=\text { const. }, \quad \zeta \in \Gamma .
$$

Conversely, if $f, F$ are holomorphic in $D$ and $D^{*}$, respectively, $F(\infty)=0$ and (2.6) holds for some real $\lambda,|\lambda|>1$, then $\lambda$ is a Fredholm eigenvalue of a sufficiently smooth $\Gamma$. In fact, both $\mu$ and $v$ can be recovered from the boundary values by means of (2.6) and then the formula (2.3) determines $f$ and $F$. This also results from Theorem 5 in [6].

In [14] Schiffer was concerned with the derivatives of holomorphic potentials of double layer rather than the potentials themselves. This resul:ed in the absence of the condition $F(\infty)=0$ and in dropping $\lambda=1$ as an eigenvalue; cf. p. 1191 in [14].

The facts just mentioned may serve as a justification for the following definitions.

Definition 1. We call $(f, F)$ a pair of conjugate holomorphic eigenfunctions (abbreviated: CHE) of a Jordan curve $\Gamma$ in $\boldsymbol{C}$ if $f$ and $F$ are non-constant functions holomorphic in the domains $D$ and $D^{*} \cup\{\infty\}$, respectively, that satisfy the following conditions:

(i) $f$ and $F$ have extensions to the closures of the respective domains which are continuous in the spherical metric and statisfy the boundary condition (2.7) on $\Gamma$;

(ii) If $h$ and $h^{*}$ map conformally the unit disk $\Delta$ onto $D$ and $D^{*}$, respectively, then for any $w \in \partial \Delta$ there exists a neighborhood $N_{w}$ of $w$ such that both $f \circ h$ and $F \circ h^{*}$ are univalent in $N_{w} \cap \Delta$. Moreover, $f \circ h$ and $F \circ h^{*}$ belong to $H^{1}(\Delta)$.

Definition 2. If the functions $f, F$ satisfy the conditions of Definition 1, then the real constant $\lambda$ determined by (2.9) and the real-valued function

$$
\mu(\zeta)=\frac{1}{2}[f(\zeta)+\overline{F(\zeta)}], \quad \zeta \in \Gamma,
$$

are called a Fredholm eigenvalue and a Fredholm eigenfunction of $\Gamma$ associated with $\lambda$, respectively.

Note that no regularity conditions are imposed on $\Gamma$ in the above-stated definitions. In what follows we shall use the term classical Fredholm eigenvalue, whenever Fredholm eigenvalues in the usual sense appear. If $F(\infty)=0$ and $\Gamma$ is sufficiently smooth, then $\lambda$ satisfying the assumptions of Definition 2 is, as mentioned above, a classical eigenvalue of $\Gamma$. 
The assumption (ii) in the Definition 1 implies finite valence of the same order for both $f$ and $F$. Moreover, due to the boundary relation (2.7) the Riemann surfaces of both CHE can be welded to a punctured sphere.

Most properties of Fredholm eigenvalues in the sense of Definition 2 are the same as in the classical case and the proofs are even much simpler in many cases. Here we list some of these properties whose proofs will be published in a separate paper to appear in the Annales Polonici Mathematici. The references that follow a property are related to the classical case.

(i) There is no Jordan curve with Fredholm eigenvalue 1, or -1 .

(ii) If $\lambda$ is a Fredholm eigenvalue of $\Gamma$, so is $-\lambda$.

(iii) All Fredholm eigenvalues of $\Gamma$ satisfy $|\lambda|>1$.

(iv) If $h$ is a Möbius transformation and the Jordan curves $\Gamma, \Gamma_{1}=h(\Gamma)$ do not contain $\infty$, then the sets of eigenvalues of $\Gamma$ and $\Gamma_{1}$ coincide; cf. [14], p. 1195.

(v) If $\lambda>1$ and one of the Dirichlet integrals $\iint_{D}\left|f^{\prime}\right|^{2}, \iint_{D^{*}}\left|F^{\prime}\right|^{2}$ is finite, the other one is also finite and we have

$$
(\lambda-1) \iint_{D}\left|f^{\prime}\right|^{2}=(\lambda+1) \iint_{D^{*}}\left|F^{\prime}\right|^{2}
$$

cf. formula (25), p. 1193 in [14].

(vi) If $\Gamma$ admits $K$-qc reflection, then Ahlfors's inequality

$$
\lambda \geqq(K+1) /(K-1)
$$

holds for all positive eigenvalues $\lambda$ corresponding to $\mathrm{CHE}$ with finite Dirichlet integral (cf. [1]).

There are relatively few Jordan curves for which Fredholm eigenvalues are known. We give two examples.

Example 1. Let $E$ be the ellipse $\xi=e^{i \theta}+k e^{-i \theta}, 0 \leqq \theta \leqq 2 \pi, 0<k<1$. Any eigenvalue of $E$ has the form $\mp k^{-n}, n=1,2, \ldots$ (cf. [14]). It is easily seen that $f(z)=(k-1)^{-1} z, \quad F(Z)=(2 k)^{-1}\left[Z-\left(Z^{2}-4 k\right)^{1 / 2}\right]$ are CHE associated with $\lambda_{1}=1 / k$. Let $P_{n}(z)$ be the sequence of polynomials defined as follows:

$$
P_{1}(z)=z, \quad P_{2}(z)=z^{2}-2 k, \quad P_{n+1}(z)=z P_{n}(z)-k P_{n-1}(z) .
$$

One can verify that $f_{n}(z)=\left(k^{n}-1\right)^{-1} P_{n}(z), \quad F_{n}(Z)=(F(Z))^{n}$ are CHE associated with $\lambda_{n}=k^{-n}$.

Example 2. Let $\Gamma$ be a circular wedge symmetric w.r.t. the real axis with vertices $-1,1$ and interior angles $\alpha \pi, 0<\alpha<1$. The functions $f(z)=$ $(1 / \alpha) \log (1+z) /(1-z), \quad F(Z)=(1 /(2-\alpha)) \log (Z-1) /(Z+1) \quad$ are $\mathrm{CHE}$ associated with the eigenvalue $\lambda=(1-\alpha)^{-1}$. 


\section{Construction of the extremal quasiconformal reflection}

We shall now prove the following

Theorem. Let $\Gamma$ be a Jordan curve in the finite plane $C$. Suppose conjugate holomorphic eigenfunctions, $f, F$ associated with a Fredholm eigenvalue $\lambda>1$ of $\Gamma$ are locally univalent in $D$ and $D^{*}$, respectively. Then $\Gamma$ admits $a K$-qc reflection $\varphi$ with $K=(\lambda+1) /(\lambda-1)$ which satisfies the relation

$$
F \circ \varphi=l \circ f \text { in } D, f \circ \varphi=L \circ F \text { in } D^{*} \text {, }
$$

where $l$ and $L$ are defined by (2.8). If $\iint_{D}\left|f^{\prime}\right|^{2}<\infty$, then $\varphi$ is unique extremal and $\lambda$ is the smallest eigenvalue corresponding to CHE with finite Dirichlet integrals.

Proof. By our assumptions the functions $f, F$ map conformally simply connected domains $D, D^{*}$ onto simply connected Riemann surfaces $S, S^{*}$ over the $w$-plane, with no branch points. The relation (2.7) induces a homeomorphic correspondence between their boundary curves. The affine mapping $l$ carries $S$ onto a homeomorphic Riemann surface $\tilde{S}$ whose projection on the $w$-plane as well as the boundary curve are the same as those of $S^{*}$ in view of (2.7). By the property (ii) of Definition 1 and the argument principle each point $w$ of the projection has the same number of preimages in both $\tilde{S}$ and $S^{*}$. We now establish a correspondence between the points of $\tilde{S}$ and $S^{*}$ with the same projection $w$ so as to obtain a resulting lifted mapping $\varphi$ of $D$ onto $D^{*}$ which is injective and satisfies $F \circ \varphi=l \circ f$ in $D$. To this end take an arbitrary path $\gamma$ in $D$ joining $\zeta \in \Gamma$ to $z \in D$. If $w_{1}=f(z)$ and $w=l\left(w_{1}\right)$, then $\tilde{\gamma}=l \circ f(\gamma)$ is a path joining in the common projection of $\tilde{S}$ and $S^{*}$ the points $F(\zeta)=l \circ f(\zeta)$ and $W=F(Z), Z=\varphi(z)$ to be determined. Due to the local univalence of $F$ there exists a unique analytic continuation of $F^{-1}$ along $\tilde{\gamma}$ with the initial value $\zeta$ which determines $F^{-1}(w)=Z=\varphi(z)$ in a unique manner. In fact, if the end-points $\zeta, z$ of $\gamma$ are fixed and $\gamma$ varies in $D$ in a continuous manner, the resulting branch of $F^{-1}$ at $w$ remains unchanged, and is thus independent of a particular choice of $\gamma$. The same argument is applied if now $\zeta$ is changing on $\Gamma$ while $z$ is fixed. Therefore $Z$ does not depend on $\zeta$ either.

The mapping $\varphi: D \rightarrow D^{*}$ is well-defined and satisfies $\varphi=F^{-1} \circ / \circ f$ locally in $D$, so it is continuous. Since $\varphi$ has a continuous extension on $\Gamma$ that satisfies $\varphi(\zeta)=\zeta, \zeta \in \Gamma$, it must be a homeomorphism by the argument principle. Thus $\varphi$ is a $K$-qc reflection in $\Gamma$ with $K=(\lambda+1) /(\lambda-1)$, which means that $\Gamma$ is a $K$-quasicircle. The $K$-qc reflection $\varphi$ can be extended to a sense-reversing $K$-qc selfmapping of $\hat{C}$ which has the form $f^{-1} \circ L \circ F$ locally in $D^{*}$. Thus $\varphi$ is the homeomorphic solution of the Beltrami type equation

$$
\frac{\varphi_{z}}{\varphi_{\bar{z}}}=\left\{\begin{array}{rc}
-(1 / \lambda) f^{\prime}(z) / \overline{f^{\prime}(z),}, & z \in D, \\
(1 / \lambda) F^{\prime}(z) / \overline{F^{\prime}(z)}, & z \in D^{*},
\end{array}\right.
$$

that keeps the points of $\Gamma$ fixed. 
We now prove that $\varphi$ is the unique extremal qc reflection in $\Gamma$ as soon as $\iint_{D}\left|f^{\prime}\right|^{2}$ is finite. Let $g$ be a conformal mapping of $D$ onto the unit disk $\Delta$ and let $G$ be a conformal mapping of $D^{*}$ onto $C \backslash \bar{\Delta}=\Delta^{*}$. Let $\sigma$ denote the reflection in $\partial \Delta$. If $\Phi$ is any reflection in $\Gamma$ and $h=\sigma \circ G \circ \varphi \circ g^{-1}$, then $H=\sigma \circ G \circ \Phi \circ g^{-1}$ belongs to the class $Q_{h}$ of qc selfmappings of $\Delta$ with fixed boundary values determined by $h$ and studied by Reich and Strebel; cf. e.g. [13]. Obviously the maximal dilatations of $\Phi$ and $H$ are equal. Complex dilatation $\varrho(w)$ of $h$ satisfies

$$
\varrho(w)=-\frac{1}{\lambda} \overline{\left(\frac{f^{\prime}(z)}{g^{\prime}(z)}\right)} /\left(\frac{f^{\prime}(z)}{g^{\prime}(z)}\right), \quad z=g^{-1}(w) .
$$

Putting $\psi(w)=\left[i f^{\prime}(z) / g^{\prime}(z)\right]^{2}, \quad z=g^{-1}(w)$, we obtain $\varrho(w)=(1 / \lambda) \overline{\psi(w)} /|\psi(w)|$ so $h$ is a Teichmüller mapping for which $\iint_{\Delta}|\psi|=\iint_{D}\left|f^{\prime}\right|^{2}<\infty$. Therefore, according to Strebel [15], $h$ and also $\varphi$ are unique extremal.

Suppose now that there exists an eigenvalue $\lambda_{1}$ with $1<\lambda_{1}<\lambda$, where $f, F$ are CHE associated with $\lambda$ and satisfy our assumptions. Since $\Gamma$ admits the $K$-qc reflection $\varphi$ just described with $K=(\lambda+1) /(\lambda-1)$, we have $\lambda=(K+1) /(K-1)$ and hence $\lambda_{1} \geqq \lambda$ by (2.12), which is a contradiction. This ends the proof.

Corollary 1. There is an obvious connection between qc reflections $\Phi$ in $\Gamma$ (with transfinite diameter $d(\Gamma)=1$ ) and functions of the familiar class $\Sigma(\chi) ; c f$. [8]. If $h \in \Sigma(x)$, then $h \circ \sigma \circ h^{-1}$ is a qc reflection in $\Gamma=h(\partial \Delta)$. Conversely, given conformal mapping $h$ of $\Delta$ onto the inside of a Jordan curve $\Gamma$ and a qc reflection $\Phi$ in $\Gamma$, a qc extension of $h$ on $\Delta^{*}$ is given by $\Phi \circ h \circ \sigma$.

Corollary 2. If one of the CHE $f, F$ is univalent, the other one is also univalent and the corresponding eigenvalue $\lambda>1$ is the smallest one. Moreover, $\varphi=F^{-1} \circ l \circ f$ in $D, \varphi=f^{-1} \circ L \circ F$ in $D^{*}$.

This result is essentially equivalent to Theorem 7 in [8], in view of Corollary 1. We end by giving the following

Example 3. Consider the analytic Jordan curve

$$
\Gamma: \zeta=e^{i \theta}\left(1+k e^{-3 i \theta}\right)^{2 / 3}, \quad 0 \leqq \theta \leqq 2 \pi,
$$

where $0<k<1$. Kühnau has shown in [7] that $\lambda>1 / k$, where $\lambda$ is the smallest eigenvalue of $\Gamma$. However by Corollary 1 and [7], the extremal reflection in $\Gamma$ has maximal dilatation equal to $(1+k) /(1-k)>(\lambda+1) /(\lambda-1)$. Hence by the Theorem, locally univalent $\mathrm{CHE}$ associated with $\Gamma$ do not exist. Thus even for an analytic Jordan curve with smallest eigenvalue $\lambda>1$, it is not always possible to construct a $K$-quasiconformal reflection in $\Gamma$ with $K=(\lambda+1) /(\lambda-1)$. 


\title{
References
}

[1] Ahlfors, L. V.: Remarks on the Neumann-Poincaré integral equation. - Pacific J. Math. 2, 1952, 271-280.

[2] GaIER, D.: Konstruktive Methoden der konformen Abbildung. - Springer-Verlag, BerlinGöttingen-Heidelberg, 1964.

[3] Gehring, F. W.: Characteristic properties of quasidisks. - Les Presses de l'Université de Montréal, Montréal, 1982.

[4] Golusin, G. M.: Geometrische Funktionentheorie. - VEB Deutscher Verlag der Wissenschaften, Berlin, 1957.

[5] KellogG, O. D.: Foundations of potential theory. - Verlag von Julius Springer, Berlin, 1929.

[6] KÜHNAU, R.: Eine Integralgleichung in der Theorie der quasikonformen Abbildungen. - Math. Nachr. 76, 1977, 139-152.

[7] KüHNAU, R.: Zu den Grunskyschen Koeffizientenbedingungen. - Ann. Acad. Sci. Fenn. Ser. A I Math. 6, 1981, 125-130.

[8] KÜHNAU, R.: Quasikonforme Fortsetzbarkeit, Fredholmsche Eigenwerte und Grunskysche Koeffizientenbedingungen. - Ann. Acad. Sci. Fenn. Ser. A I Math. 7, 1982, 383-391.

[9] Lehto, O.: Quasiconformal mappings in the plane. - Lecture Note 14, University of Maryland, College Park, Maryland, 1975, 1-43.

[10] Lehto, O., and K. I. VIRTANEN: Quasiconformal mappings in the plane. - Springer-Verlag, Berlin-Heidelberg-New York, 1973.

[11] LovirT, W. V.: Linear integral equations. - Dover Publ., New York, 1950.

[12] Petrovsky, I. G.: Lectures on partial differential equations. - Interscience Publishers, Inc., New York-London, 1954.

[13] Reich, E., and K. StREBel: Extremal quasiconformal mappings with given boundary values. Contributions to Analysis, A collection of papers dedicated to Lipman Bers, edited by L. V. Ahlfors et al. Academic Press, New York-London, 1974, 375-391.

[14] SCHIFFer, M.: The Fredholm eigenvalues of plane domains. - Pacific J. Math. 7, 1957, 11871225.

[15] Strebel, K.: Zur Frage der Eindeutigkeit extremaler quasikonformer Abbildungen des Einheitskreises II. - Comment. Math. Helv. 39, 1964, 77-89.

\author{
Uniwersytet Marii Curie-Skłodowskiej \\ Instytut Matematyki \\ Ul. M. Curie-Skłodowskiej 1 \\ 20-031 Lublin \\ Poland
}

Received 21 November 1983 